Article

\title{
Giant Enhancement of THz Wave Emission under Double-Pulse Excitation of Thin Water Flow
}

\author{
Hsin-hui Huang ${ }^{1}$, Takeshi Nagashima ${ }^{2, *}$ (i) , Tetsu Yonezawa ${ }^{3,4}$ (D), Yasutaka Matsuo ${ }^{5}$, \\ Soon Hock Ng 6 (iD), Saulius Juodkazis $6,7,8, *$ (i) and Koji Hatanaka 1,9,10,* (iD) \\ 1 Research Center for Applied Sciences, Academia Sinica, Taipei 11529, Taiwan; hsinhuih@gate.sinica.edu.tw \\ 2 Faculty of Science and Engineering, Setsunan University, 17-8 Ikeda-Nakamachi, \\ Neyagawa, Osaka 572-8508, Japan \\ 3 Division of Materials Science and Engineering, Faculty of Engineering, Hokkaido University, \\ Hokkaido 060-8628, Japan; tetsu@eng.hokudai.ac.jp \\ 4 Institute for the Promotion of Business-Regional Collaboration, Hokkaido University, Hokkaido 001-0021, Japan \\ 5 Research Institute for Electronic Science, Hokkaido University, Hokkaido 001-0021, \\ Japan; matsuo@es.hokudai.ac.jp \\ 6 Centre for Micro-Photonics and ARC Training Centre in Surface Engineering for Advanced Materials (SEAM), \\ School of Science, Swinburne University of Technology, Hawthorn, VIC 3122, \\ Australia; soonhockng@swin.edu.au \\ 7 World Research Hub Initiative (WRHI), School of Materials and Chemical Technology, \\ Tokyo Institute of Technology, 2-12-1 Ookayama, Meguro-ku, Tokyo 152-8550, Japan \\ 8 Institute of Advanced Sciences, Yokohama National University, 79-5 Tokiwadai, \\ Hodogaya-ku, Yokohama 240-8501, Japan \\ 9 College of Engineering, Chang Gung University, Taoyuan 33302, Taiwan \\ 10 Department of Materials Science and Engineering, National Dong-Hwa University, Hualien 97401, Taiwan \\ * Correspondence: t-nagash@mpg.setsunan.ac.jp (T.N.); sjuodkazis@swin.edu.au (S.J.); \\ kojihtnk@gate.sinica.edu.tw (K.H.); Tel.: +81-72-813-1167 (T.N.); +61-3-9214-8718 (S.J.); +886-2-2787-3132 (K.H.)
}

Received: 4 February 2020; Accepted: 12 March 2020; Published: 17 March 2020

\begin{abstract}
Simultaneous measurements of $\mathrm{THz}$ wave and hard X-ray emission from thin and flat water flow when irradiated by double femtosecond laser pulses $(800 \mathrm{~nm}, 35 \mathrm{fs} /$ transform-limited, $0.5 \mathrm{kHz}$, delay times up to $15 \mathrm{~ns}$ ) were carried out. THz wave measurements by time-domain spectroscopy and X-ray detection by Geiger counters were performed at the transmission and the reflection sides of the flow. THz wave emission spectra show their dynamic peak shifts toward the low frequency with the highest intensity enhancements more than $1.5 \times 10^{3}$ times in $|\boldsymbol{E}|^{2}$ accumulated over the whole spectrum range of $0-3 \mathrm{THz}$ at the delay time of $4.7 \mathrm{~ns}$ between the two pulses. On the other hand, X-ray intensity enhancements are limited to about 20 times at $0 \mathrm{~ns}$ under the same experimental conditions. The mechanisms for the spectral changes and the intensity enhancements in $\mathrm{THz}$ wave emission are discussed from the viewpoint of laser ablation on the water flow induced by the pre-pulse irradiation.
\end{abstract}

Keywords: femtosecond laser; intense laser; water; THz wave; X-ray; time-domain spectroscopy; ablation; double-pulse excitation; plasma; intensity enhancement 


\section{Introduction}

$\mathrm{THz}$ science and technology [1] have been continuously growing since the early report on $\mathrm{THz}$ wave emission [2]. Applications have been demonstrated in imaging [3] with optical component developments [4], spectroscopy for mesoscopic structures [5], coherent measurements of intense $\mathrm{THz}$ wave [6], gas-detection in Fabry-Pérot cavity [7], tunable/compact laser [8], sensing for medical uses [9], and next generation 6G wireless communication [10]. Recent progress includes studies under scanning tunneling microscopy [11-13] with nanoscale spatial resolution. For further developments in the field, innovations in $\mathrm{THz}$ wave sources are expected.

One of the experimental methods for $\mathrm{THz}$ wave emission, apart from the well-developed techniques with semiconductors such as ZnTe and others [14], is through femtosecond laser-based plasma formation [15] since much higher incident laser intensity can be put into the plasma formation. While solid samples are irreversibly damaged under such intense laser irradiation conditions, air [16,17] and noble gases (He, $\mathrm{Ne}, \mathrm{Ar}, \mathrm{Kr}$, and $\mathrm{Xe}$ ) [18,19] have been used as targets for plasma-based $\mathrm{THz}$ emission, thus circumventing the issue. Laser excitation with chirped pulses [20] or two-colors [16,20-23] has been carried out. The mechanisms of the THz wave emission have been discussed mainly in terms of four-wave mixing and ponderomotive force. Furthermore, a feedback method of laser wave-front manipulation can control THz wave emission [24]. One disadvantage of gaseous targets is low absorption cross section at the incident laser wavelength. Considering the damage issue in solid samples, liquids can be an ideal target for plasma-based $\mathrm{THz}$ wave emission since it can be refreshed continuously during experiments. Indeed, experiments with a water film [25-29] or a line [30] and other liquids such as deionized-water, alcohols, acetone, dichloroethane, or carbon disulfide [31] have been carried out. It is also reported that $\mathrm{THz}$ wave emission can be induced in association with X-ray emission from thin water flow and its intensity is enhanced under double-pulse excitation [32].

Water, among various liquids, is considered to be one of the best targets for intense laser irradiation for $\mathrm{THz}$ wave emission without serious degradation. Even at high laser repetition rates, liquid target can be used. Indeed, experiments with distilled water and other solutions were reported recently as described, discussions started on the emission mechanisms and characteristics of THz source. Similar to THz wave emission at meV-photon energies, $\mathrm{X}$-ray emission at $\mathrm{keV}$ from water and aqueous solutions with solutes or additives such as electrolytes $[33,34]$ and gold nano-particles $[35,36]$ has been also reported. Water as a universal solvent is a good target for investigation of mechanisms and for development of $\mathrm{THz}$ wave sources for bright emission.

Double-pulse excitation is another method for the THz wave emission enhancements [32,37-39]. Under double-pulse excitation of Ar gas at delay times of 500 ps, THz emission increases 10-times as compared with single-pulse excitation [37]. In the case of flat water flow, when the thickness of jet was 100-240 $\mu \mathrm{m}$ at the delay time of $\sim 6 \mathrm{ps}$, THz emission increases 4-times [39], while in the case of water jet of $210 \mu \mathrm{m}$ diameter at the delay time of $50 \mathrm{ps}$, THz pulse energy increases more than 8-times [38]. For thinner flat water flow with $20 \mu \mathrm{m}$ thickness, the enhancement was about 100-times in $|\boldsymbol{E}|^{2}$ estimated from the signal peak intensities in time-domain spectroscopy at the nanosecond delays [32]. As the related-mechanisms have been already discussed in X-ray emission from aqueous solutions [33,40], the pre-pulse irradiation plays an important role in pre-plasma formation and in dynamic morphological changes due to ablation within picosecond to nanosecond time delays. Water surface can be controlled on-demand by pre-pulse irradiation for plasma formation leading to solvated electron formation in water [41] and for transient surface structure formation by ablation [33]. However, better understanding of the detailed mechanisms of $\mathrm{THz}$ wave emission and its enhancement are still indispensable especially in the field of strongly localized light-matter interactions occurring at focal volumes from nanoscale comparable 
with the wavelength of the incident laser pulses to micro-scale comparable with the wavelength of resultant $\mathrm{THz}$ wave emission.

In this paper, we report simultaneous measurements for $\mathrm{THz}$ wave and $\mathrm{X}$-ray emission from thin water flow under double-pulse excitation with delay times up to $15 \mathrm{~ns}$. Finely synchronized THz wave and X-ray radiation is required for their complimentary usage, for instance, for imaging [42]. This study explores the enhancement of $\mathrm{THz}$ wave radiation from the simultaneous $\mathrm{THz} / \mathrm{X}$-ray emission source, which was first reported in gas, glass, and water [32,43,44].

\section{Experimental Setup}

\subsection{Double-Pulse Excitation of Water Flow}

Figure 1a shows the experimental setup. The water flow nozzle system with two pipette tips and a reservoir, which has been introduced previously [46], was used for creating a thin water flow. The flow thickness was measured at $17 \pm 2 \mu \mathrm{m}$ with a laser-based thickness measurement system ( $0.25 \mu \mathrm{m}$ resolution, SI-T80, Keyence, Osaka, Japan). The width of the flow is $8 \mathrm{~mm}$ at most, which is much wider than the laser focus size. More than $120 \mathrm{~mL}$ of water was being circulated by a pump (PMD-211, SANSO, Hyogo, Japan) with the flow rate at $70 \mathrm{~mL} / \mathrm{min}$ during experiments. The estimated flow velocity at the laser focus is $5.8 \mathrm{~m} / \mathrm{s}$, which means that the water target moves about 1-cm in every laser irradiation and refreshes its surface. The flow angle and its position were controlled mechanically. The water flow position was monitored by a laser-based displacement sensor ( $0.2 \mu \mathrm{m}$ resolution, LK-G80, Keyence, Osaka, Japan).

A pulsed femtosecond laser ( $>35 \mathrm{fs} /$ transform-limited, $800 \mathrm{~nm}, \mathrm{TEM}_{00}, \mathrm{M}^{2}<1.3$, the contrast ratio $>1000: 1$ to the pre-pulse and $>100: 1$ to the post pulse, Mantis, Legend Elite HE USP, Coherent, Inc., Santa Clara, CA, USA) was used. The laser beam was split into the pre-pulse (vertically/s- pol., $0.1 \mathrm{~mJ} /$ pulse) and the main pulse (horizontally/p- pol.). The remaining main pulse was split into the probe for THz time-domain spectroscopy (THz-TDS) and the main pulse $(0.4 \mathrm{~mJ} /$ pulse) with a series of half-wave plates and polarization beam splitters (65-906, 47-048, Edmund Optics, Barrington, NJ, USA). The time delay between the pre-pulse and the main pulse was determined to be from $0 \mathrm{~ns}$ up to $15 \mathrm{~ns}$ using tunable optical delays, and these two pulses were combined co-linearly for the double-pulse excitation using a cubic beam splitter (47-048, Edmund Optics, Barrington, NJ, USA). This combined beam passed through an optical chopper (3502, New Focus, CA, USA) which reduced the repetition rate from $1 \mathrm{kHz}$ to $0.5 \mathrm{kHz}$ for the lock-in measurements. The combined beams were tightly focused with a 1-inch off-axis parabolic mirror (OAPM, effective focal length $f=50.8 \mathrm{~mm}, 47-097$, Edmund Optics, Barrington, NJ, USA) onto the thin water film with the incident angle of 60 degrees to the flow normal. 


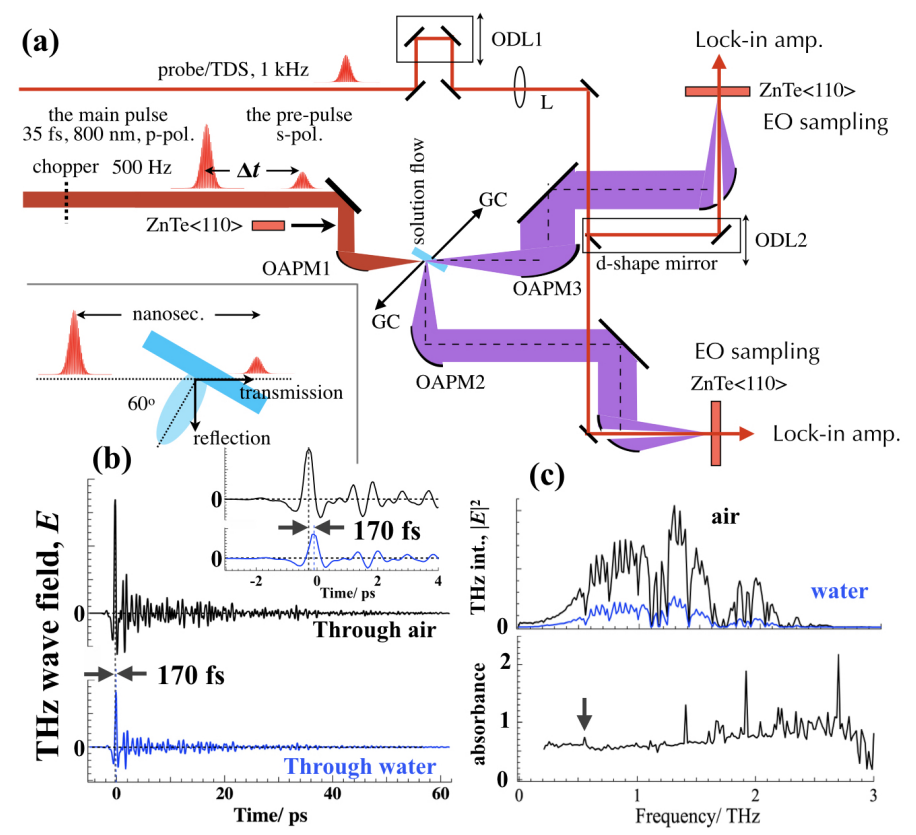

Figure 1. (a) The experimental setup for $\mathrm{THz}$ wave with $\mathrm{X}$-ray detections in the transmission and the reflection directions with time-domain spectroscopy (TDS). ODL1, optical delay for TDS. ODL2, optical delay for time matching between the transmission and the reflection directions. L, plano-convex lens $(f=50 \mathrm{~cm})$. The thickness of $\langle 110\rangle$-oriented ZnTe crystal for TDS was $1 \mathrm{~mm}$. The parent focal lengths for the off-axis parabolic mirrors (OAPMs) were $f=50.8 \mathrm{~mm}$ (OAPM1, 1-inch diameter), $101.6 \mathrm{~mm}$ (OAPM2, 2-inch diameter), $152.4 \mathrm{~mm}$ (OAPM3, 2-inch diameter), and two $101.6 \mathrm{~mm}$ (2-inch diameter with a hole in its center for the probe) respectively. GC, Geiger counter. The inset shows the geometrical configuration of the flow, the laser incidence, and ablation phenomena in the nanosecond time delay. (b) TDS signals from a 1-mm thick $\langle 110\rangle$-oriented ZnTe crystal though air and the water flow at 60 degrees to the laser incident angle. The inset represents the TDS signals near the zero delay. (c) Emission spectra in the THz frequency calculated from the TDS signals (the top) and calculated absorption spectrum of the water flow as $\log \left(|E|_{\text {air }}{ }^{2} /|E|_{\text {water }}{ }^{2}\right)$ (the bottom). The arrow indicates the absorption peak at $0.56 \mathrm{THz}$ [45].

\subsection{THz Wave Time-Domain Spectroscopy in the Transmission/Reflection Directions}

$\mathrm{THz}$ wave emission in the reflection and the transmission directions, which are the front side and the back side of the flow, respectively, was recorded simultaneously by EO sampling with a $\langle 110\rangle$-oriented ZnTe crystal (1-mm thick, Nippon Mining \& Metals Co., Ltd. Tokyo, Japan). This setup is an update from the previous one [32], which has shortened the experimental time much. Briefly, the emission was collected by an OAPM ( $f=101.6 \mathrm{~mm}$, MPD249-M01, ThorLabs, Newton, NJ, USA) in reflection and by another OAPM ( $f=152.4 \mathrm{~mm}$, MPD269-M01, ThorLabs, Newton, NJ, USA) in transmission. The probe for TDS was split into two beams spatially with a d-shaped mirror (PFD05-03-M01, ThorLabs, Newton, NJ, USA) which was set on a manual stage (ODL2) in order to match the time zero between the reflection and the transmission directions. The difference of the detection efficiencies in the reflection and the transmission directions was calibrated as follows; THz emission from a 1-mm thick $\langle 110\rangle$-oriented ZnTe crystal set before the OAPM1 was split into the transmission and the reflection components by an undoped silicon thin film as a THz beam splitter or bent by a conventional gold mirror placed at the water flow position. By measuring the TDS signal of THz wave in each direction, the difference of the detection efficiencies 
in the two directions was calibrated. With this system setup using the 1-mm thick $\langle 110\rangle$-oriented ZnTe crystals as detectors for EO sampling, the detectable frequency range extends to $\sim 3 \mathrm{THz}$ at the highest [14].

\subsection{X-ray Detection}

Geiger counters (SS315, Southern Scientific, West Sussex, UK) with calibrated sensitivity were used for X-ray measurements at the front and the back sides as described in detail elsewhere [35,40]. The Geiger counters were set at $15^{\circ}$ to the flow normal and $12 \mathrm{~cm}$ away from the water flow during the experiments. The vertically polarized pre-pulse intensity in this experiment is low at $0.1 \mathrm{~mJ} /$ pulse and its irradiation does not induce detectable X-ray emission since the interaction of s-pol. light with the self-generating plasma is much less effective than the case with p-pol. light [47,48]. All the experiments in this paper were carried out in air under atmospheric pressure (1 atm) at room temperature (296 K). Therefore, it is certain that the Geiger counter detects only X-ray, not $\alpha$ - or $\beta$-rays.

\section{Results}

\section{1. $\mathrm{THz}$ Spectroscopy}

First, TDS measurements of THz wave emission from a 1-mm thick <110>-oriented ZnTe crystal set before the OAPM1 through the water flow were carried out. The residual laser pulse after the crystal was blocked by a black poly-propylene sheet placed right after the crystal. Figure $1 \mathrm{~b}$ shows the TDS waveforms through the water flow and air (without the flow). Here, the TDS signals were obtained from -5 ps to 61.6 ps every 0.03 ps. The water flow displays a time shift, $\Delta t_{w}=170 \pm 0.6 \mathrm{fs}$, as compared with air. With the refractive index of water at $1 \mathrm{THz}, n_{\text {water }}^{\mathrm{THz}}=2.12$ [49] and the light velocity in water, $v_{\text {water }}=c / n=1.4 \times 10^{8} \mathrm{~m} / \mathrm{s}$, considering the incident angle of 60 degrees to the flow normal, the thickness of the water flow is estimated to be $d_{w}=14 \pm 0.1 \mu \mathrm{m}$. The water absorption spectrum as $\log \left(|\boldsymbol{E}|_{\text {air }}{ }^{2} /|\boldsymbol{E}|_{\text {water }}{ }^{2}\right)$ shown in the bottom of Figure 1c is calculated with discrete Fourier-transform of the TDS signals shown in the top of Figure 1c. With the absorption intensity at $1 \mathrm{THz}$ and the known absorption coefficient [49], the flow thickness is estimated to be $15.7 \mu \mathrm{m}$, which is well matched with the estimated values within experimental errors. In the spectrum, there are a few sharp lines (e.g., indicated by an arrow at $0.56 \mathrm{THz}$ ), which can be assigned to water monomer rotational energy levels [50]. The flow thickness estimated is much smaller than the wavelength in the $\mathrm{THz}$ wave emission $(\sim 300 \mu \mathrm{m})$, therefore interference due to internal multiple reflection in the flow as considered in the previous report [51] is negligible.

Figure 2a,b shows normalized spectra of $\mathrm{THz}$ emission in transmission and reflection from the water flow irradiated by the laser pluses. Here, the experimental condition is slightly different from that in Figure $1 \mathrm{~b}$; the TDS signals were obtained from $-10 \mathrm{ps}$ to 24 ps every $0.07 \mathrm{ps}$. 

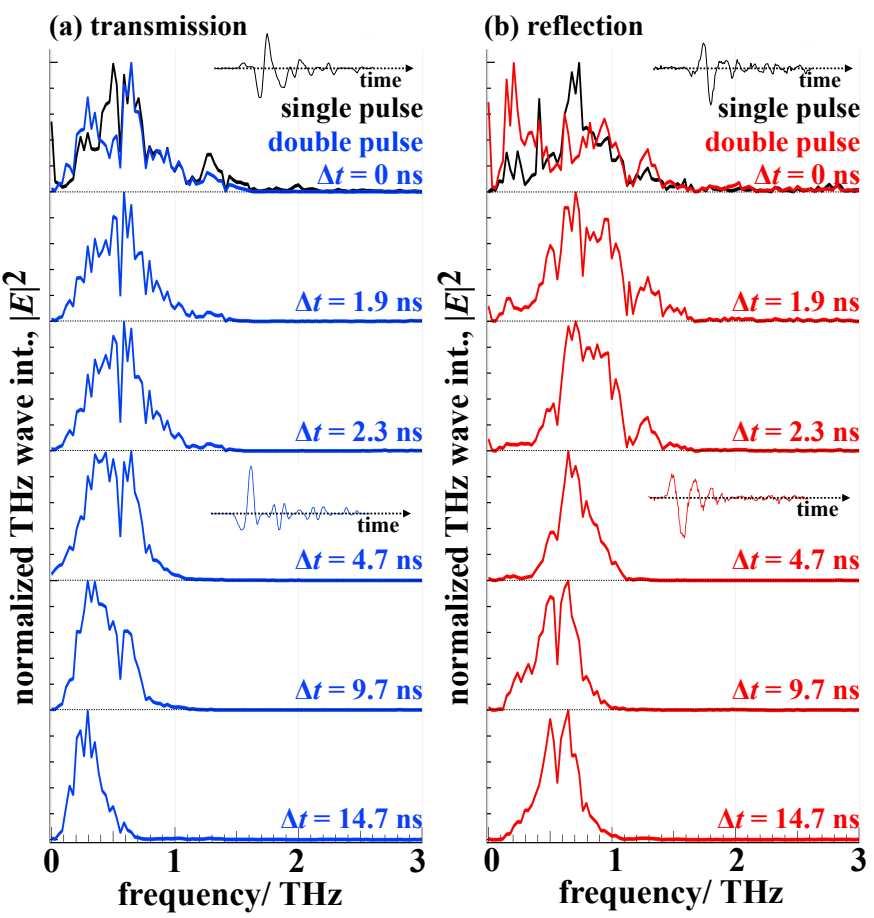

Figure 2. Normalized THz emission spectra in (a) the transmission and (b) the reflection sides under the single pulse (in black on the top) or the double-pulse excitation conditions. The vertical axes represent $\mathrm{THz}$ wave $|\boldsymbol{E}|^{2}$ intensity. The insets in the single-pulse excitation case and $\Delta t=4.7 \mathrm{~ns}$ represent the original signal waveforms from -3 ps to 7 ps in TDS measurements.

Under the single-pulse excitation condition, the spectra are similar to each other in both of the directions; the peak frequency is at around $0.5-0.6 \mathrm{THz}$ and the tail extends to $1.5 \mathrm{THz}$. Under the double-pulse excitation condition, the series of the spectra commonly show their dynamic peak shifts toward the lower frequency in the nanosecond time range (to the maximum delay at $14.7 \mathrm{~ns}$ ). In this time range, after the decay of the plasma by the pre-pulse irradiation, laser ablation phenomena such as transient surface roughness with ripple formation, shock-wave expansion, and micro-droplet/mist ejection in the direction parallel to the flow normal as shown in the inset of Figure 1a [52] are already initiated. This is reconfirmed in the observation of the water absorption peak at $0.56 \mathrm{THz}$ (the arrow in Figure 1c) even for the reflection direction (Figure $2 b$ ).

\section{2. $\mathrm{THz}$ Enhancement}

Figure 3a-d shows dynamic changes of the enhancement factors of the total $\mathrm{THz}$ wave emission in $|\boldsymbol{E}|^{2}$ estimated over the frequency range in $0-3 \mathrm{THz}$ and X-ray emission normalized to the emission intensity under the single-pulse irradiation condition in transmission and reflection, respectively. In the previous report [32], we estimated such enhancement factor only based on the peak intensity of the original TDS waveform as $\boldsymbol{E}$-field intensity. However, the estimation in the current manner is more reliable considering all the frequency components. Furthermore, we have confirmed that the same enhancement factors are obtained even from the data in the previous paper [32]. With the pre-pulse (s-pol.) and the main pulse (p-pol.) intensities at $0.1 \mathrm{~mJ} /$ pulse and $0.4 \mathrm{~mJ} /$ pulse, respectively, and the transform-limited pulse at $35 \mathrm{fs}$, the enhancement of the THz emission shows its highest at the 2-5 ns window, while that of 
the X-ray shows its peak at $0 \mathrm{~ns}$. Evidently, this data set shows the different enhancement mechanisms for both emissions.
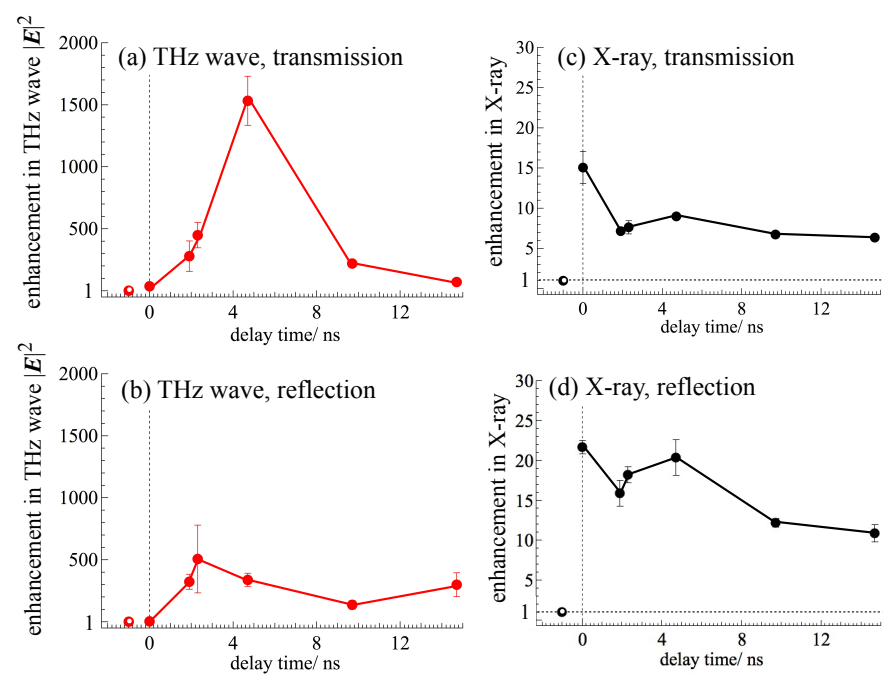

Figure 3. Enhancements in $\mathrm{THz}$ wave emission in $|\boldsymbol{E}|^{2}(\mathbf{a}, \mathbf{b})$ and X-ray $(\mathbf{c}, \mathbf{d})$ in the transmission and the reflection sides as a function of the delay time between the pre-pulse and the main-pulse irradiations. The vertical axes are normalized with the emission intensity under the single-pulse excitation condition (open circles).

In the case of X-ray, the pre-pulse irradiation induces the plasma formation and its fast decay at around 0 ns delay time, then the main pulse irradiates this pre-excited region leading to jump in plasma temperature and electron density. High temperature is the cause of $X$-ray emission and its enhancement [33]. In the case of $\mathrm{THz}$ emission which may depend on temperature gradient [53,54], such hot plasma formation may interfere with THz emission since the critical density of plasma [55], $n_{c}=\omega_{T H z}^{2} m_{e} \varepsilon_{0} / e^{2}$, where $n_{c}$, $\omega_{T H z}, \varepsilon_{0}, m_{e}$ and $e$ are the critical electron density, THz frequency, permittivity, electron mass and charge, respectively, makes the excited region opaque. In the nanoseconds time delay after the pre-pulse irradiation, various laser ablation phenomena described above have already started. The acoustic space-time scaling of sub- $\mu \mathrm{m}$ features formed in a few tens of $\mu$ s as described above. Importantly, the highest enhancement in $\mathrm{THz}$ wave emission under the double-pulse excitation is $1.5 \times 10^{3}$ times in intensity $|\boldsymbol{E}|^{2}$ at 4.7 ns delay in the transmission direction, while the X-ray enhancement is only 15-22 times at the highest.

\section{Discussion}

\subsection{Effects of the Pre-Pulse Irradiation}

By considering the ablation phenomena at nanoseconds time delays after the pre-pulse, the observed dynamic spectral shift toward the low frequency and the giant enhancement of $\mathrm{THz}$ emission are discussed from the viewpoints of $\mathrm{THz}$ wave absorption by water, surface structural modifications of the flow such as roughness/ripple formation, mist ejection, and swelling, an elongation of $\mathrm{THz}$ wave source, and $\mathrm{THz}$ wave emission characteristics.

Based on the knowledge of laser ablation of liquids under the similar experimental conditions [52], the structural modifications of the water flow induced by the pre-pulse irradiation, nano- to micro-scale roughness/ripples, droplet/mist ejection, and pressure wave propagation with the swelling on the rear surface are considered to be shown in Figure 4a. The scale length is governed by the speed of sound $v_{s}=1.481 \mathrm{~km} / \mathrm{s}$ (under normal conditions) and the delay time after the pre-pulse irradiation, $\Delta t$. At the 
delay time of $4.7 \mathrm{~ns}$, for instance, such pressure wave travels $v_{s} \times \Delta t \approx 7 \mu \mathrm{m}$, which is comparable with the thickness of the water flow. Hence, the pressure induces the increase of mass density $+\Delta \rho$ due to the compression and causes the rarefaction $-\Delta \rho$ at the central part which leads to cavitation on longer time scale.

\subsection{Spectral Peak Shift}

One straightforward idea is that droplet/mist ejection in the detection path for the $\mathrm{THz}$ wave emission in the transmission and the reflection directions may cause additional absorption for $\mathrm{THz}$ wave emission as shown in Figure $4 \mathrm{~b}$. The water absorption spectrum shows its higher intensity at the higher frequency as reported previously [56], which will cause an apparent peak shift toward the lower frequency as the droplet/mist ejection takes place.

Second, interferometric effects for $\mathrm{THz}$ wave emission due to the transient surface roughness/ripple formation on the flow surface are considered to be shown in Figure $4 \mathrm{~b}$. It was reported that laser excitation of $\mathrm{LiNbO}_{3}$ by transient grating controls $\mathrm{THz}$ emission spectra within the range of $0.5-3 \mathrm{THz}$ [57]. Similar studies with InAs proved that periodic structures of $\mathrm{THz}$ wave emission source show the peak shift toward the lower frequency as the period increases from $190 \mu \mathrm{m}$ to $360 \mu \mathrm{m}$ [58]. Under the current experimental condition for the pre-pulse irradiation to induce laser ablation, transient surface roughness is considered to be composed of different orders of spatial frequencies from a few tens of nano-meters to micrometers [52]. Such surface structures can cause the dynamic spectral shift under the delayed main-pulse irradiation.

\subsection{Giant Enhancement in $\mathrm{THz}$ Emission}

As shown in Figure 3c,d, X-ray intensity, not only THz wave emission, also increases to some extent at the same delay time of $4.7 \mathrm{~ns}$. These enhancements in the both emissions in $\mathrm{THz}$ wave and X-ray indicate that the absorption of the main-pulse increases effectively and that the density and the temperature of electrons increases in the self-generating plasma by the main-pulse irradiation. The pre-pulse irradiation induces the droplet/mist formation and its expansion outward with time. Then, the delayed main pulse induces the self-generating plasma with a larger volume in the expanding droplet/mist, which results in the enhanced absorption. Or, due to the flow surface with the structural modulations such as nano-to micro-scale roughness/ripples induced by the pre-pulse irradiation, the effective absorption efficiency of the main pulse increases through multiple scatterings. Such dynamic morphological changes keep growing even after $4.7 \mathrm{~ns}$ and become larger in size and inappropriate for $\mathrm{THz}$ enhancements. X-ray generation is primarily dependent on the peak temperature of electrons, $T_{e}$, while $\mathrm{THz}$ emission can be dependent on its transient $\mathrm{d} T_{e} / \mathrm{d} t[53,54]$. Both entities are decaying on longer times causing lower emission of X-rays and $\mathrm{THz}$ wave, respectively.

In summary, we tentatively consider that the giant $\mathrm{THz}$ wave enhancement at $4.7 \mathrm{~ns}$ is attributed to the self-generating plasma with the larger volume in the expanding droplet/mist induced by the pre-pulse irradiation. In addition, the modification of the rear surface induced by the pre-pulse irradiation as shown in Figure 4 may contribute to the enhancement observed in the transmission side at the delay time of $4.7 \mathrm{~ns}$. To reach more quantitative descriptions of the light-matter interaction for the described experiment, a fast optical imaging will be added to reveal the geometry and its changes during the laser ablation of the water flow by the pre-pulse irradiation. For the complete mechanism clarification, plasma dynamics diagnostics and/or simulations on hydrodynamics are also indispensable as future work. Knowledge of formation dynamics of wave guide-like channel with density gradient in the flow (prepared by the pre-pulse irradiation) for efficient coupling between the main pulse and the flow and on extent of nano-to 
micro-droplets/mist and swelling is necessary to quantify and further validate separate contributions of discussed mechanisms to the enhancement of $\mathrm{THz}$ wave emission.
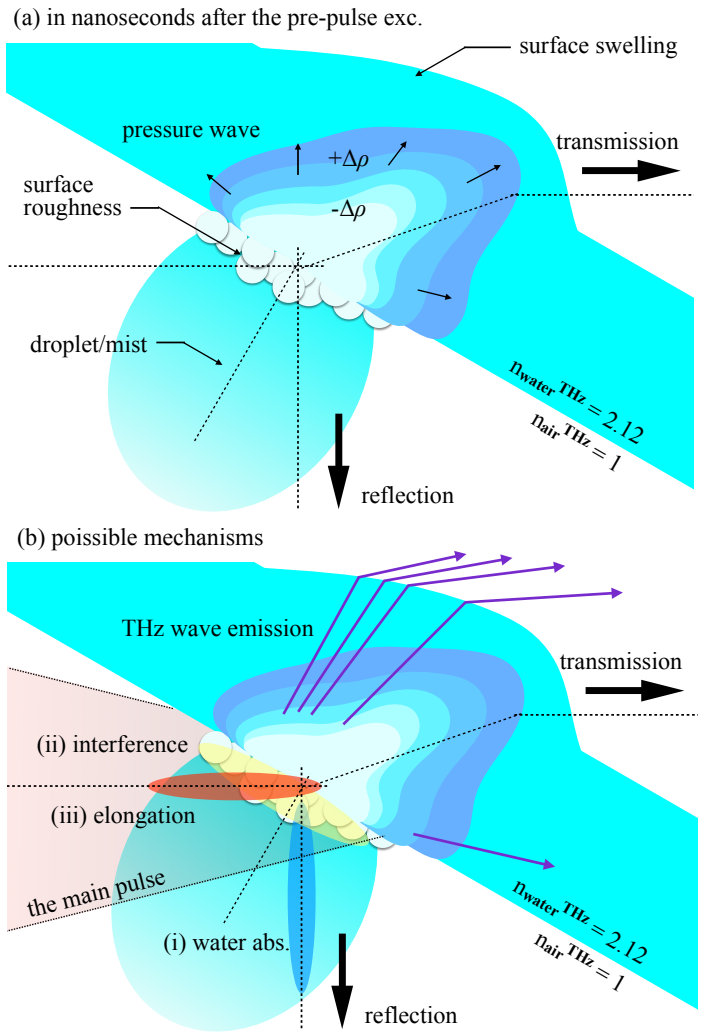

Figure 4. (a) A schematic diagram for the water flow structure (top view) at the delay time in nanoseconds after the pre-pulse irradiation. (b) Possible mechanisms for the interaction of the main pulse with the structured water flow and resultant $\mathrm{THz}$ wave emission.

\section{Conclusions and Outlook}

This paper describes the simultaneous emission of $\mathrm{THz}$ wave and hard X-ray from distilled water when it is irradiated by focused double pulse of femtosecond laser. THz wave emission spectra show their dynamic peak shifts toward the lower frequency, which has been discussed in the viewpoints of laser ablation induced by the pre-pulse irradiation. THz wave emission intensity $|\boldsymbol{E}|^{2}$ increases more than $1.5 \times 10^{3}$ times when it is compared to the emission intensity under the single-pulse excitation condition, which is recalculated to be 10-times higher in $|\boldsymbol{E}|^{2}$ intensity than the THz wave emission from the conventional $\langle 110\rangle$-oriented ZnTe crystal. Further enhancements are surely expected with chirped pulse excitation $[20,59]$ or with the addition of solutes [60,61]. Optimization of the double-pulse irradiation and target solutions will be focus of next studies for advancements of $\mathrm{THz}$ sources.

Author Contributions: H.-h.H. and K.H. performed experiments. H.-h.H., T.N., S.J., and K.H. analyzed the data and wrote the manuscript. Y.M. supplied the Si beam splitter and edited the manuscript. T.Y. and S.H.N. joined the discussions and edited the manuscript. All authors have read and agreed to the published version of the manuscript.

Funding: T.N. is grateful for the support by JSPS KAKENHI Grant Number 17K05086. T.Y. is grateful for the support by Grant-in-Aid for Scientific Research for Fostering Joint International Research (B) (18KK0159). S.J. is grateful for the support via the Australian Research Council Discovery project DP190103284. K.H. is grateful for the supports by the Ministry of Science and Technology (MOST) of Taiwan (107-2112-M-001-014-MY3), the Cooperative Research Program of "Network Joint Research Center for Materials and Devices", Nanotechnology Platform, and 
the Collaborative Research Projects of Laboratory for Materials and Structures, Institute of Innovative Research, Tokyo Institute of Technology.

Conflicts of Interest: The authors declare no conflict of interest. The founding sponsors had no role in the design of the study; in the collection, analyses or interpretation of data; in the writing of the manuscript; nor in the decision to publish the results.

\section{References}

1. Mittleman, D.M. Perspective: Terahertz science and technology. J. Appl. Phys. 2017, 122, 230901. [CrossRef]

2. Fattinger, C.; Grischkowsky, D. Terahertz beams. Appl. Phys. Lett. 1989, 54, 490-492. [CrossRef]

3. Mittleman, D.M. Twenty years of terahertz imaging. Opt. Express 2018, 26, 9417-9431. [CrossRef]

4. Sahoo, A.; Yang, C.S.; Yen, C.L.; Lin, H.C.; Wang, Y.J.; Lin, Y.H.; Wada, O.; Pan, C.L. Twisted nematic liquid-crystal-based terahertz phase shifter using pristine PEDOT: PSS transparent conducting electrodes. Appl. Sci. 2019, 9, 761. [CrossRef]

5. Li, R.; D'Agostino, C.; McGregor, J.; Mantle, M.D.; Zeitler, J.A.; Gladden, L.F. Mesoscopic structuring and dynamics of alcohol/water solutions probed by terahertz time-domain spectroscopy and pulsed field gradient nuclear magnetic resonance. J. Phys. Chem. B 2014, 118, 10156-10166. [CrossRef]

6. Mou, S.; Rubano, A.; Paparo, D. Terahertz hyper-Raman time-domain spectroscopy of gallium selenide and its application in terahertz detection. Appl. Phys. Lett. 2019, 115, 211105. [CrossRef]

7. Hindle, F.; Bocquet, R.; Pienkina, A.; Cuisset, A.; Mouret, G. Terahertz gas phase spectroscopy using a high-finesse Fabry-Pérot cavity. Optica 2019, 6, 1449-1454. [CrossRef]

8. Chevalier, P.; Amirzhan, A.; Wang, F.; Piccardo, M.; Johnson, S.G.; Capasso, F.; Everitt, H.O. Widely tunable compact terahertz gas lasers. Science 2019, 366, 856-860. [CrossRef]

9. Sun, C.K.; Chen, H.Y.; Tseng, T.F.; You, B.; Wei, M.L.; Lu, J.Y.; Chang, Y.L.; Tseng, W.L.; Wang, T.D. High sensitivity of T-ray for thrombus sensing. Sci. Rep. 2018, 8, 3948. [CrossRef]

10. Yang, P.; Xiao, Y.; Xiao, M.; Li, S. 6G Wireless communications: Vision and potential techniques. IEEE Net. 2019, 33, 70-75. [CrossRef]

11. Cocker, T.L.; Jelic, V.; Gupta, M.; Molesky, S.J.; Burgess, J.A.J.; Reyes, G.D.L.; Titova, L.V.; Tsui, Y.Y.; Freeman, M.R.; Hegmann, F.A. An ultrafast terahertz scanning tunnelling microscope. Nat. Photon. 2013, 7, 620-625. [CrossRef]

12. Klarskov, P.; Kim, H.; Colvin, V.L.; Mittleman, D.M. Nanoscale laser terahertz emission microscopy. ACS Photon. 2017, 4, 2676-2680. [CrossRef]

13. Mastel, S.; Lundeberg, M.B.; Alonso-González, P.; Gao, Y.; Watanabe, K.; Taniguchi, T.; Hone, J.; Koppens, F.H.L.; Nikitin, A.Y.; Hillenbrand, R. Terahertz nanofocusing with cantilevered terahertz-resonant antenna tips. Nano Lett. 2017, 17, 6526-6533. [CrossRef]

14. Wilke, I.; Sengupta, S. Nonlinear optical techniques for terahertz pulse generation and detection-Optical rectification and electrooptic sampling. In Terahertz Spectroscopy: Principles and Applications, 1st ed.; Dexheimer, S.L., Ed.; CRC Press: Boca Raton, FL, USA, 2007; Chapter 2, pp. 41-72. [CrossRef]

15. Hamster, H.; Sullivan, A.; Gordon, S.; Falcone, R.W. Short-pulse terahertz radiation from high-intensity-laser-produced plasmas. Phys. Rev. E 1994, 49, 671-677. [CrossRef]

16. Cook, D.J.; Hochstrasser, R.M. Intense terahertz pulses by four-wave rectification in air. Opt. Lett. 2000, $25,1210-1212$. [CrossRef]

17. Andreeva, V.A.; Kosareva, O.G.; Panov, N.A.; Shipilo, D.E.; Solyankin, P.M.; Esaulkov, M.N.; González de Alaiza Martínez, P.; Shkurinov, A.P.; Makarov, V.A.; Bergé, L.; et al. Ultrabroad terahertz spectrum generation from an air-based filament plasma. Phys. Rev. Lett. 2016, 116, 063902. [CrossRef]

18. Chen, Y.; Yamaguchi, M.; Wang, M.; Zhang, X.C. Terahertz pulse generation from noble gases. Appl. Phys. Lett. 2007, 91, 251116. [CrossRef]

19. Mori, K.; Hashida, M.; Nagashima, T.; Li, D.; Teramoto, K.; Nakamiya, Y.; Inoue, S.; Sakabe, S. Increased energy of THz waves from a cluster plasma by optimizing laser pulse duration. AIP Adv. 2019, 9, 015134.

20. Zhang, Z.; Panov, N.; Andreeva, V.; Zhang, Z.; Slepkov, A.; Shipilo, D.; Thomson, M.D.; Wang, T.J.; Babushkin, I.; Demircan, A.; et al. Optimum chirp for efficient terahertz generation from two-color femtosecond pulses in air. Appl. Phys. Lett. 2018, 113, 241103. [CrossRef] 
21. Kress, M.; Löffler, T.; Eden, S.; Thomson, M.; Roskos, H.G. Terahertz-pulse generation by photoionization of air with laser pulses composed of both fundamental and second-harmonic waves. Opt. Lett. 2004, 29, 1120-1122. [CrossRef]

22. Zhong, H.; Karpowicz, N.; Zhang, X.C. Terahertz emission profile from laser-induced air plasma. Appl. Phys. Lett. 2006, 88, 261103. [CrossRef]

23. Babushkin, I.; Kuehn, W.; Köhler, C.; Skupin, S.; Bergé, L.; Reimann, K.; Woerner, M.; Herrmann, J.; Elsaesser, T. Ultrafast spatiotemporal dynamics of terahertz generation by ionizing two-color femtosecond pulses in gases. Phys. Rev. Lett. 2010, 105, 053903. [CrossRef]

24. Hah, J.; Jiang, W.; He, Z.H.; Nees, J.A.; Hou, B.; Thomas, A.G.R.; Krushelnick, K. Enhancement of THz generation by feedback-Optimized wavefront manipulation. Opt. Express 2017, 25, 17271-17279. [CrossRef]

25. Tcypkin, A.N.; Ponomareva, E.A.; Putilin, S.E.; Smirnov, S.V.; Shtumpf, S.A.; Melnik, M.V.; E, Y.; Kozlov, S.A.; Zhang, X.C. Flat liquid jet as a highly efficient source of terahertz radiation. Opt. Express 2019, 27, 15485-15494. [CrossRef]

26. Tcypkin, A.N.; Melnik, M.V.; Zhukova, M.O.; Vorontsova, I.O.; Putilin, S.E.; Kozlov, S.A.; Zhang, X.C. High kerr nonlinearity of water in THz spectral range. Opt. Express 2019, 27, 10419-10425. [CrossRef]

27. Qi, J.; Yiwen, E.; Williams, K.; Dai, J.; Zhang, X.-C. Observation of broadband terahertz wave generation from liquid water. Appl. Phys. Lett. 2017, 111, 071103. [CrossRef]

28. E, Y.; Jin, Q.; Tcypkin, A.; Zhang, X.C. Terahertz wave generation from liquid water films via laser-induced breakdown. Appl. Phys. Lett. 2018, 113, 181103. [CrossRef] [PubMed]

29. Jin, Q.; Dai, J.; E, Y.; Zhang, X.C. Terahertz wave emission from a liquid water film under the excitation of asymmetric optical fields. Appl. Phys. Lett. 2018, 113, 261101. [CrossRef] [PubMed]

30. Zhang, L.L.; Wang, W.M.; Wu, T.; Feng, S.J.; Kang, K.; Zhang, C.L.; Zhang, Y.; Li, Y.T.; Sheng, Z.M.; Zhang, X.C. Strong terahertz radiation from a liquid-water line. Phys. Rev. Appl. 2019, 12, 014005. [CrossRef]

31. Dey, I.; Jana, K.; Fedorov, V.Y.; Koulouklidis, A.D.; Mondal, A.; Shaikh, M.; Sarkar, D.; Lad, A.D.; Tzortzakis, S.; Couairon, A.; et al. Highly efficient broadband terahertz generation from ultrashort laser filamentation in liquids. Nat. Commun. 2017, 8, 1184. [CrossRef]

32. Huang, H.H.; Nagashima, T.; Hsu, W.H.; Juodkazis, S.; Hatanaka, K. Dual THz wave and X-ray generation from a water film under femtosecond laser excitation. Nanomaterials 2018, 8, 523.

33. Hatanaka, K.; Ono, H.; Fukumura, H. X-ray pulse emission from cesium chloride aqueous solutions when irradiated by double-pulsed femtosecond laser pulses. Appl. Phys. Lett. 2008, 93, 064103. [CrossRef]

34. Hatanaka, K.; Miura, T.; Fukumura, H. Ultrafast x-ray pulse generation by focusing femtosecond infrared laser pulses onto aqueous solutions of alkali metal chloride. Appl. Phys. Lett. 2002, 80, 3925-3927. [CrossRef]

35. Masim, F.C.P.; Porta, M.; Hsu, W.H.; Nguyen, M.T.; Yonezawa, T.; Balčytis, A.; Juodkazis, S.; Hatanaka, K. Au nanoplasma as efficient hard X-ray emission source. ACS Photon. 2016, 3, 2184-2190. [CrossRef]

36. Hsu, W.H.; Masim, F.C.P.; Balčytis, A.; Huang, H.H.; Yonezawa, T.; Kuchmizhak, A.A.; Juodkazis, S.; Hatanaka, K. Enhancement of X-ray emission from nanocolloidal gold suspensions under double-pulse excitation. Beilstein J. Nanotechnol. 2018, 9, 2609-2617. [CrossRef]

37. Mori, K.; Hashida, M.; Nagashima, T.; Li, D.; Teramoto, K.; Nakamiya, Y.; Inoue, S.; Sakabe, S. Directional linearly polarized terahertz emission from argon clusters irradiated by noncollinear double-pulse beams. Appl. Phys. Lett. 2017, 111, 241107. [CrossRef] [PubMed]

38. E, Y.; Jin, Q.; Zhang, X.C. Enhancement of terahertz emission by a preformed plasma in liquid water. Appl. Phys. Lett. 2019, 115, 101101. [CrossRef]

39. Ponomareva, E.A.; Tcypkin, A.N.; Smirnov, S.V.; Putilin, S.E.; Yiwen, E.; Kozlov, S.A.; Zhang, X.C. Double-pump technique-One step closer towards efficient liquid-based THz sources. Opt. Express 2019, $27,32855-32862$. [CrossRef]

40. Hsu, W.H.; Masim, F.C.P.; Balčytis, A.; Juodkazis, S.; Hatanaka, K. Dynamic position shifts of X-ray emission from a water film induced by a pair of time-delayed femtosecond laser pulses. Opt. Express 2017, 25, 24109-24118. [CrossRef] 
41. Bruggeman, P.J.; Kushner, M.J.; Locke, B.R.; Gardeniers, J.G.E.; Graham, W.G.; Graves, D.B.; Hofman-Caris, R.C.H.M.; Maric, D.; Reid, J.P.; Ceriani, E.; et al. Plasma-liquid interactions: a review and roadmap. Plasma Sour. Sci. Technol. 2016, 25, 053002. [CrossRef]

42. Zhang, X.C.; Shkurinov, A.; Zhang, Y. Extreme terahertz science. Nat. Photon. 2017, 11, 16-18. [CrossRef]

43. Balakin, A.V.; Dzhidzhoev, M.S.; Gordienko, V.M.; Esaulkov, M.; Zhvaniya, I.A.; Ivanov, K.A.; Kotelnikov, I.; Kuzechkin, N.A.; Ozheredov, I.A.; Panchenko, V.Y.; et al. Interaction of high-intensity femtosecond radiation with gas cluster beam: Effect of pulse duration on joint terahertz and X-ray emission. IEEE Trans. Terahertz Sci. Tech. 2016, 7, 70-79.

44. Hamster, H.; Sullivan, A.; Gordon, S.; White, W.; Falcone, R.W. Subpicosecond, electromagnetic pulses from intense laser-plasma interaction. Phys. Rev. Lett. 1993, 71, 2725-2728. [CrossRef]

45. Exter, M.V.; Fattinger, C.; Grischkowsky, D. Terahertz time-domain spectroscopy of water vapor. Opt. Lett. 1989, 14, 1128-1130. [CrossRef]

46. Hsu, W.H.; Masim, F.C.P.; Porta, M.; Nguyen, M.T.; Yonezawa, T.; Balčytis, A.; Wang, X.; Rosa, L.; Juodkazis, S.; Hatanaka, K. Femtosecond laser-induced hard X-ray generation in air from a solution flow of Au nano-sphere suspension using an automatic positioning system. Opt. Express 2016, 24, 19994-20001. [CrossRef]

47. Attwood, D. Soft X-Rays and Extreme Ultraviolet Radiation; Cambridge University Press: Cambridge, UK, 1999. [CrossRef]

48. Turcu, I.C.E.; Dance, J.B. X-Rays from Laser Plasmas; WILEY: Hoboken, NJ, USA, 1998. [CrossRef]

49. Zhou, J.; Rao, X.; Liu, X.; Li, T.; Zhou, L.; Zheng, Y.; Zhu, Z. Temperature dependent optical and dielectric properties of liquid water studied by terahertz time-domain spectroscopy. AIP Adv. 2019, 9, 035346. [CrossRef] [PubMed]

50. Xin, X.; Altan, H.; Saint, A.; Matten, D.; Alfano, R.R. Terahertz absorption spectrum of para and ortho water vapors at different humidities at room temperature. J. Appl. Phys. 2006, 100, 094905. [CrossRef] [PubMed]

51. Jin, Q.; E, Y.; Gao, S.; Zhang, X.C. Investigation of liquid lines as terahertz emitters under ultrashort optical excitation. arXiv e-prints 2019, arXiv:1902.07150. [CrossRef]

52. Hatanaka, K.; Tsuboi, Y.; Fukumura, H.; Masuhara, H. Nanosecond and femtosecond laser photochemistry and ablation dynamics of neat liquid benzenes. J. Phys. Chem. B 2002, 106, 3049-3060.

53. Oladyshkin, I.V. Diagnostics of the electron scattering in metals in terms of a terahertz response to femtosecond laser pulse. JETP Lett. 2016, 103, 435-439.

54. Ilyakov, I.E.; Shishkin, B.V.; Fadeev, D.A.; Oladyshkin, I.V.; Chernov, V.V.; Okhapkin, A.I.; Yunin, P.A.; Mironov, V.A.; Akhmedzhanov, R.A. Terahertz radiation from bismuth surface induced by femtosecond laser pulses. Opt. Lett. 2016, 41, 4289-4292. [CrossRef]

55. Chen, F.F. Introduction to Plasma Physics and Controlled Fusion; Springer US: New York, NY, USA, 1984. [CrossRef]

56. $\mathrm{Xu}$, J; Plaxco, K.W.; Allen, S.J. Absorption spectra of liquid water and aqueous buffers between 0.3 and $3.72 \mathrm{THz}$. J. Chem. Phys. 2006, 124, 036101.

57. Stepanov, A.G.; Hebling, J.; Kuhl, J. Generation, tuning, and shaping of narrow-band, picosecond THz pulses by two-beam excitation. Opt. Express 2004, 12, 4650-4658. [CrossRef]

58. Hiratsuka, M.; Nagashima, T.; Hangyo, M. Tunable THz radiation from semiconductor surfaces irradiated by optical pulses with interference pattern. J. Jpn. Soc. Infrared Sci. Technol. 2007, 16, 56-61. [CrossRef]

59. Hatanaka, K.; Ida, T.; Ono, H.; Matsushima, S.; Fukumura, H.; Juodkazis, S.; Misawa, H. Chirp effect in hard X-ray generation from liquid target when irradiated by femtosecond pulses. Opt. Express 2008, 16, 12650-12657. [CrossRef] [PubMed] 
60. Hatanaka, K.; Miura, T.; Fukumura, H. White X-ray pulse emission of alkali halide aqueous solutions irradiated by focused femtosecond laser pulses: A spectroscopic study on electron temperatures as functions of laser intensity, solute concentration, and solute atomic number. Chem. Phys. 2004, 299, 265-270.

61. Huang, H.H.; Chau, Y.T.R.; Yonezawa, T.; Nguyen, M.T.; Zhu, S.; Deng, D.; Nagashima, T.; Hatanaka, K. $\mathrm{THz}$ wave emission from ZnTe nano-colloidal aqueous dispersion irradiated by femtosecond laser. Chem. Lett. 2020. [CrossRef]

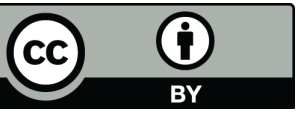

(C) 2020 by the authors. Licensee MDPI, Basel, Switzerland. This article is an open access article distributed under the terms and conditions of the Creative Commons Attribution (CC BY) license (http:/ / creativecommons.org/licenses/by/4.0/). 